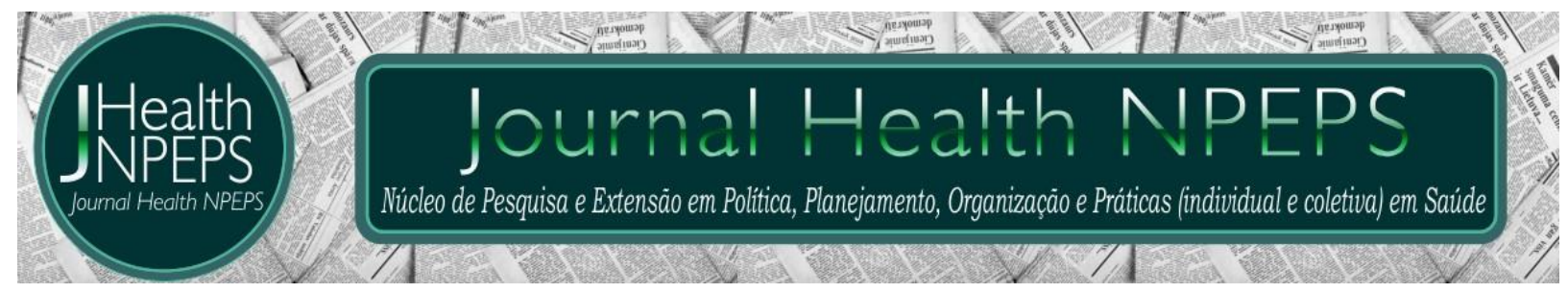

http: / /dx.doi.org/10.30681/252610102775

ARTIGO ORIGINAL

\title{
Identificação de fatores de risco para apneia obstrutiva do sono após adenotonsilectomia: estudo piloto
}

\section{Identification of risk factors for obstructive sleep apnea after adenotonsilectomy: pilot study}

\section{Identificación de factores de riesgo para apneia obstructiva del sueño después adenotonsilectomía: estudio piloto}

\section{Larissa Pierri Carvalho ${ }^{1}$, Renato Oliveira Martins ${ }^{2}$, Eduardo Leite Fonseca ${ }^{3}$, Camila de Castro Corrêa ${ }^{4}$, Silke Anna Theresa Weber ${ }^{5}$}

\section{RESUMO}

Objetivo: identificar possíveis fatores de risco para a ocorrência de distúrbios respiratórios obstrutivos graves no pós-operatório imediato da adenotonsilectomia - AT. Método: trata-se de um estudo descritivo, prospectivo e de caráter observacional, realizado em 2014. Participaram crianças em rotina cirúrgica de AT. A avaliação consistiu no questionário respondido pelos pais (dados pessoais e clínicos), avaliação clínica da criança (oroscopia) e o exame de polissonografia (antes e após a AT). Resultados: foram avaliadas 30 crianças com queixas de obstrução nasal $(73,33 \%)$, respiração oral $(96,66 \%)$ e ronco $(100 \%)$. Na avaliação clínica, observou-se palato em ogiva $(75,86 \%)$ e hipertrofia das tonsilas palatinas de grau 3 (62\%). Antes da cirurgia, $40 \%$ da amostra foi diagnosticada com AOS grave, e, após a cirurgia, 10\% apresentou AOS grave. Houve tendência para o grau da hipertrofia das tonsilas, tabagismo passivo, IVAS (infecções das vias aéreas superiores) e fácies adenoideana se correlacionarem com a AOS grave. Conclusão: a polissonografia demonstrou elevada frequência de AOS grave prévia à AT, além de elevada frequência de eventos respiratórios obstrutivos após a cirurgia com potencial para complicações respiratórias.

Descritores: Apneia Obstrutiva do Sono; Tonsilectomia; Complicações Pós-Operatórias; Crianças; Sono.

\section{ABSTRACT}

${ }^{1}$ Médica. Residente em Dermatologia pela Universidade Estadual Paulista "Júlio de Mesquita Filho" (UNESP), Faculdade de Medicina de Botucatu. Botucatu - São Paulo - Brasil. E-mail: larissapcarvalho9@gmail.com ORCID ID: https: / /orcid.org/0000-0002-2261-8114

${ }^{2}$ Médico. Doutor. Universidade Estadual Paulista "Júlio de Mesquita Filho" (UNESP), Faculdade de Medicina de Botucatu. Botucatu - São Paulo - Brasil. E-mail: renatoceres@yahoo.com.br ORCID ID: https://orcid.org/0000-00021637-685X.

${ }^{3}$ Médico. Graduado pela Universidade Estadual Paulista "Júlio de Mesquita Filho" (UNESP), Faculdade de Medicina de Botucatu. Botucatu - São Paulo - Brasil. E-mail: fonseca.eduardo10@gmail.com ORCID ID: https://orcid.org/00000002-3249-8312

${ }^{4}$ Fonoaudióloga. Mestre em Ciências. Universidade Estadual Paulista "Júlio de Mesquita Filho" (UNESP), Faculdade de Medicina de Botucatu. Botucatu - São Paulo - Brasil. E-mail: camila.ccorrea@hotmail.com ORCID ID: https: / / orcid.org/0000-0001-5460-3120

${ }^{5}$ Médica. Livre docente. Universidade Estadual Paulista "Júlio de Mesquita Filho" (UNESP), Faculdade de Medicina de Botucatu. Botucatu - São Paulo - Brasil. E-mail: silke@fmb.unesp.br ORCID ID: https://orcid.org/0000-0003-3194-3039. Autor principal - Endereço para correspondência: Distrito de Rubião Júnior, S/N, 18618-970 - Botucatu - SP. 
Objective: to identify possible risk factors for the occurrence of severe obstructive respiratory disorders in the immediate postoperative period of adenotonsillectomy AT. Method: in a descriptive prospective study we invited children with indication of $A T$, held in 2014. Demographic data and clinical symptoms were collected, beside data of tonsil size, craniofacial configurations. All children realized a polysomnography the night before surgery and the night after. Results: 30 children were included, who presented complaints nasal obstruction (73.33\%), mouth breathing (96.66\%) and snoring (100\%). Hypertrophy of the tonsil size 3 was observed in $62 \%$, high palate in $75.86 \%$. Before surgery, in $40 \%$ of the children had the diagnosis of severe OSA, in $10 \%$ severe OSA was seen after surgery. There was a tendency of association of severe OSA to hypertrophy of the tonsils, passive tabagism, infections of UA and adenoid face. Conclusion: based on polysmnopraphy before surgery, a high frequency of severe OSA was diagnosed. At post-operative polysomnography, severe obstructive apneas were observed in a high percentage, which may induce to respiratory complications after surgery.

Descriptors: Obstructive Sleep Apnea; Tonsillectomy; Postoperative Complications; Child; Sleep.

\section{RESUMEN}

Objetivo: identificar posibles factores de riesgo para la ocurrencia de disturbios respiratorios obstructivos graves en el postoperatorio inmediato de la adenotonsilectomía - AT. Método: se trata de un estudio descriptivo, prospectivo y de carácter observacional, realizado en 2014. Participaron niños en rutina quirúrgica de AT. La evaluación consistió en el cuestionario respondido por los padres (datos personales y clínicos), evaluación clínica del niño (oroscopia) y el examen de polisomnografía (antes y después de la AT). Resultados: se evaluaron 30 niños con quejas de obstrucción nasal $(73,33 \%)$, respiración oral $(96,66 \%)$ y ronquido $(100 \%)$. En la evaluación clínica, se observó palato en ojiva $(75,86 \%)$ e hipertrofia de las amigdalinas palatinas de grado 3 (62\%). Antes de la cirugía, el 40\% de la muestra fue diagnosticada con AOS grave, y después de la cirugía, el $10 \%$ presentó AOS grave. Se observó una tendencia al grado de la hipertrofia de las amigdalitas, el tabaquismo pasivo, IVAS (infecciones de las vías aéreas superiores) y las fáciles adenoideanas se correlacionan con la AOS grave. Conclusión: la polisomnografía mostró una elevada frecuencia de AOS grave previa a la $A T$, además de una elevada frecuencia de eventos respiratorios obstructivos después de la cirugía con potencial para complicaciones respiratorias.

Descriptores: Apnea Obstructiva del Sueño; Tonsilectomía; Complicaciones Posoperatorias; Niño; Sueño.

\section{INTRODUÇÃO}

A Apneia Obstrutiva do Sono (AOS) é caracterizada por um aumento da resistência da via aérea superior, originada principalmente pela hipertrofia adenotonsilar e acomete cerca de $4 \%$ da população pediátrica ${ }^{1,2}$. Tem sido relacionada a alterações importantes, que não se limitam a problemas cardiovasculares ${ }^{3-5}$ e atraso do 
crescimento pondero-estatural ${ }^{6}$, mas também à pior qualidade de vida ${ }^{7}$, comprometimento na atenção ${ }^{8}$, rendimento escolar ${ }^{9}$ e na função cognitiva global ${ }^{10}$.

A adenotonsilectomia (AT) é considerada padrão ouro para o tratamento da AOS pediátrica $^{11}$. A AT representa a segunda cirurgia otorrinolaringológica mais comum em crianças, com aproximadamente 480.000 casos ambulatoriais realizados em 2010 nos Estados Unidos da América ${ }^{12}$. Sabe-se que em 1978 havia 0\% de indicação de AT $^{13}$, avançando em 2005 para aproximadamente 77\% dos casos de obstrução de vias aéreas superiores ${ }^{14}$.

Contudo, a AT não é isenta de riscos e pode ocasionar hemorragias, desidratação, náuseas, vômitos e dor ${ }^{15,16}$. Estudos apontam que a AOS grave pode ser um fator de risco substancial para o desenvolvimento de complicações cardiorrespiratórias após cirurgia ${ }^{17,18}$. E devido à escassez de diretrizes e estudos baseados em evidências que permitam uma melhor prática clínica para a atual variedade de cuidados para este grupo de pacientes, este estudo visou identificar possíveis fatores de risco para a ocorrência de distúrbios respiratórios obstrutivos graves no pós-operatório imediato da adenotonsilectomia.

\section{MÉTODOS}

Trata-se de um estudo randomizado descritivo, prospectivo e de caráter observacional, realizado no ano de 2014. Foram convidadas a participar da presente pesquisa, todas as crianças que realizaram caso novo, no ambulatório de Distúrbio do Sono de um serviço público de referência no interior do estado de São Paulo, e que apresentaram indicação de AT. Como critérios de exclusão, foram excluídas crianças que apresentaram obesidade mórbida (score Z > 3 desvios), desordens neurológicas, anomalias cromossômicas (Down, Turner), desordens neuromusculares ou uso de drogas depressoras do sistema respiratório.

Os métodos foram compostos por três partes: questionário respondido pelos pais, avaliação clínica da criança pelo médico otorrinolaringologista e pelo exame de polissonografia do sono.

Foi aplicado um questionário contendo dados pessoais (idade, gênero, score Z) e clínicos, que abrangeram itens relacionados ao momento antes e depois da cirurgia de adenoamigdalectomia, sendo esses: investigação de queixas de obstrução nasal, rinite 
alérgica, histórico de asma, tabagismo passivo, respiração oral diurna/noturna, ronco intenso/moderado/ocasionais, sono agitado, irritabilidade, déficit de concentração, sudorese noturna, déficit de atenção, sonolência diurna, enurese e cianose.

Foi realizado exame otorrinolaringológico, considerando a presença/ausência de fáceis adenoideanas, palato em ogiva (altura aumentada e largura diminuída), mordida aberta (trespasse vertical negativo) e classificação de Brodsky (de 1 a 4, sendo a maior classificação correspondente ao maior grau de hipertrofia das tonsilas palatinas).

A polissonografia na noite antes do procedimento cirúrgico e na noite seguinte pós-operatória imediata, obtendo-se medidas de fluxo aéreo oral e/ou nasal, medida de esforço respiratório torácico e abdominal, medida da saturação percutânea de oxigênio $\left(\mathrm{StO}_{2}\right)$, frequência cardíaca, posição no leito e registro de ronco. Os exames realizados foram laudados segundo os critérios da Academia Americana de Medicina do Sono, considerando apneia obstrutiva queda em mais de $90 \%$ do fluxo aéreo associado a esforço respiratório, observado nas cintas tóraco-abdominais por pelo menos dois ciclos respiratórios, e hipopneia para queda maior de $50 \%$ do sinal de fluxo aéreo associado à dessaturação de oxigênio $>3 \%$. Foram discriminados para cada paciente o Índice de Apneia e Hipopneia (IAH), Índice de Apneia Obstrutiva (IAO), Índice de Hipopneia $(\mathrm{IH})$, Índice de Dessaturação do Oxigênio (IDO) e presença de ronco. Consideramos IAH > 1 e/h como diagnóstico de Síndrome da Apneia Obstrutiva do Sono (AOS), o IAO > 10 é considerado AOS grave. Medidas da saturação da oxihemoglobina Sat $02<85 \%$ também foram consideradas como de gravidade do quadro.

Após o exame de polissonografia, foram analisados os resultados das crianças com AOS grave $(I A O>10)$ antes e após a AT. Os dados foram tabelados por meio do programa Microsoft Excel $\circledast$ para a análise descritiva e correlação estatística para identificar fatores de risco, adotando nível de significância de $5 \%$.

A presente pesquisa foi aprovada pelo Comitê de Ética em Pesquisa da Faculdade de Medicina de Botucatu, UNESP, sob o número de protocolo 4336-2012.

\section{RESULTADOS}

Foram avaliadas 30 crianças entre 2 e 12 anos, com média de idade de 6,43 anos. A distribuição por gênero e idade se encontra no gráfico 1. 
Gráfico 1 - Distribuição das crianças incluídas no estudo por gênero e idade.

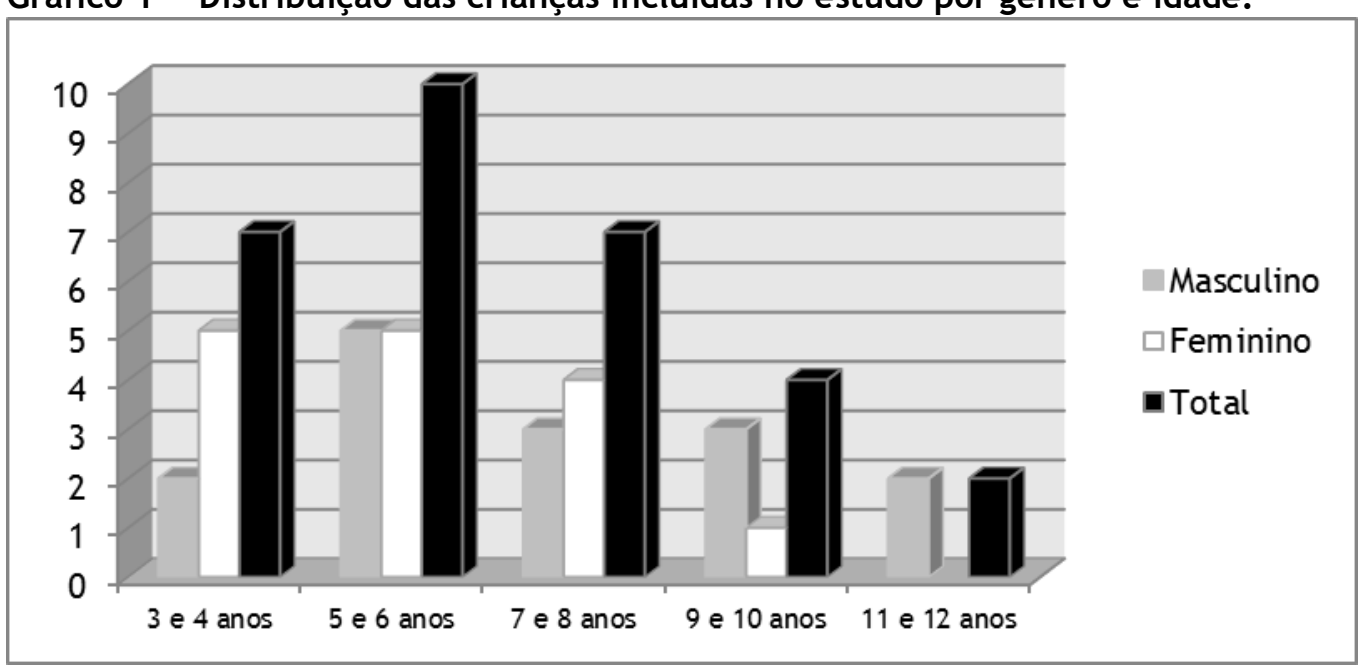

A obstrução nasal esteve presente em $73,33 \%$ dos pacientes, sendo obstrução leve em $33,33 \%$ e de moderada a grave em $40 \%$ dos casos. Oito crianças apresentaram rinite alérgica, sete como comorbidade asma e 18 exposição a tabagismo passivo.

Das 30 crianças, $96,66 \%$ eram respiradores orais sendo $36,66 \%$ respiradores orais noturnos e $60 \%$ respiradores orais noturnos e diurnos. 0 gráfico 2 demonstra a distribuição de respiradores orais por faixa etária.

Gráfico 2 - Distribuição do padrão respiratório oral por faixa etária.

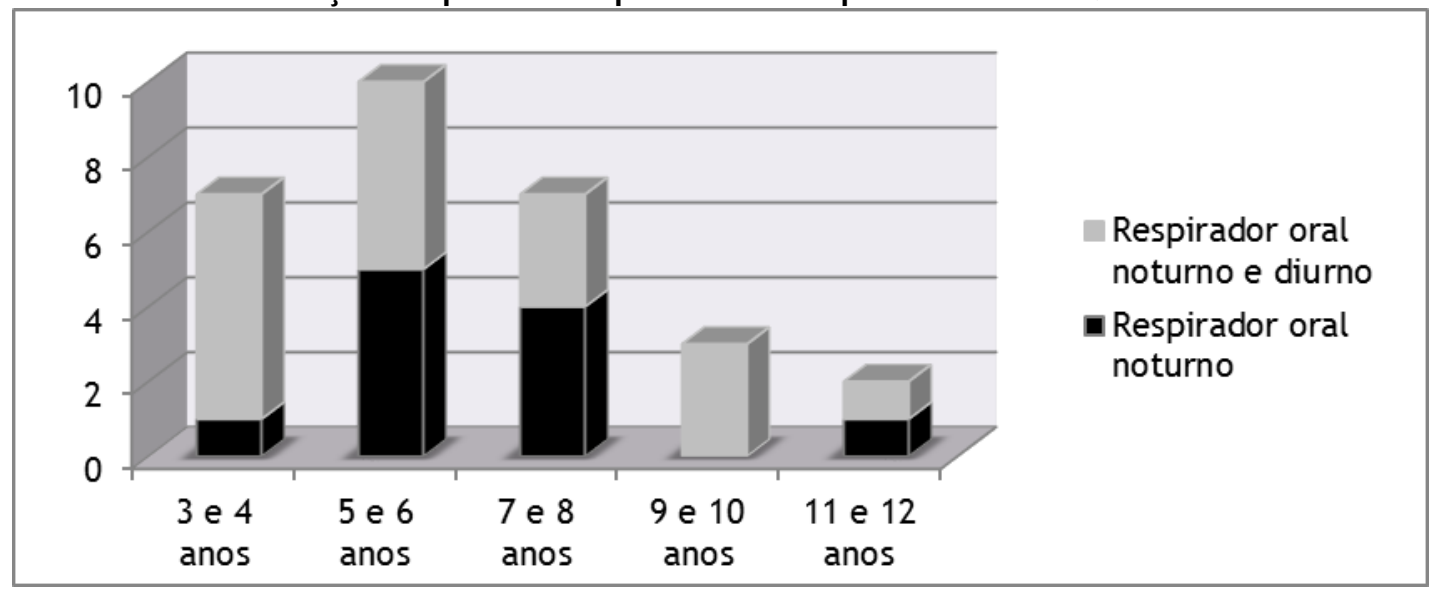

Ronco esteve presente em todas as crianças. Ronco intenso foi observado em $86,67 \%$ dos pacientes, que em sua maioria, apresentara apneia obstrutiva do sono $(84,61 \%)$. Ronco moderado foi observado em $10 \%$ dos pacientes e roncos ocasionais em $3,33 \%$. Outros sintomas constatados foram sono agitado em 28 crianças, irritabilidade por 16 , déficit de concentração por 16 , sudorese noturna em 13 crianças, déficit de atenção por 12 , sonolência diurna por seis, enurese por seis e cianose em cinco crianças. 
$\mathrm{Na}$ avaliação clínica, foi observada fácies adenoideana em19 crianças, palato em ogiva em 75,86\%, sendo que 77,27\% dessas também apresentavam fácies adenoideana. 0 palato em ogiva esteve presente associado à mordida aberta em 9 casos, palato em ogiva sem mordida aberta em 13 crianças e palato com altura/largura adequadas com mordida aberta em 1 caso.

Para o grau de hipertrofia das tonsilas segundo a classificação de Brodsky, em $24,3 \%$ das crianças foram observadas tonsilas de grau 2, 62\% com grau 3 e 13,7\% com grau 4.

Doze crianças (40\%) foram diagnosticadas com AOS grave antes da cirurgia, apresentado principalmente os seguintes fatores: exposição a tabagismo passivo, infecções de vias aéreas por repetição e rinite alérgica. Em relação à avaliação clínica, apresentaram hipertrofia de tonsilas palatinas grau 3 ou 4, fácies adenoideana e palato em ogiva. No pós-operatório imediato, $90 \%$ das crianças ainda apresentaram AOS, sendo que $10 \%$ apresentou AOS grave, embora essas não correspondessem ao grupo de AOS grave pré-operatório. Como fatores com tendência a significância observamos asma, IVAS (infecções das vias aéreas superiores) e fácies adenoideana. A tabela 1 mostra os resultados em relação aos fatores de risco para a obstrução respiratória.

Tabela 1 - Fatores de risco para AOS, AOS grave e persistência de AOS grave após a cirurgia.

\begin{tabular}{|c|c|c|c|c|c|c|c|c|c|c|c|}
\hline & $<4 \mathrm{~A}$ & $M$ & OB & TP & RA & ASMA & IVAS & B $3 / 4$ & FA/PO & EN & SD \\
\hline AOS grave pré-op & $33 \%$ & $50 \%$ & $8,3 \%$ & $66 \%$ & $41,6 \%$ & $0 \%$ & $66 \%$ & $81 \%$ & $58,3 \%$ & $25 \%$ & $33 \%$ \\
\hline AOS grave pós-op & $0 \%$ & $50 \%$ & $0 \%$ & $50 \%$ & $50 \%$ & $100 \%$ & $100 \%$ & $50 \%$ & $100 \%$ & $50 \%$ & $0 \%$ \\
\hline AOS total & $23 \%$ & $50 \%$ & $16,6 \%$ & $60 \%$ & $26,6 \%$ & $23,3 \%$ & $80 \%$ & $73 \%$ & $66 \%$ & $20 \%$ & $20 \%$ \\
\hline
\end{tabular}

Nota: op= operatório; $A=$ anos; $M=$ sexo masculino; $O B=$ obesidade; $T P=$ tabagismo passivo; $R A=$ rinite alérgica; IVAS= Infecções das Vias Aéreas Superiores; B= Brodsky; FA/PO= fácies adenoideana e palato em ogiva; $\mathrm{EN}=$ enurese noturna; $\mathrm{SD}=$ sonolência excessiva diurna.

A estatística não mostrou algum fator correlacionar significantemente com a AOS grave $(p<0,05)$ para nenhum dos fatores, porém mostrou tendência de significância $(0,05<p<0,10)$ para tonsilas 3 e 4 , tabagismo passivo, IVAS e fácies adenoideana.

Nenhuma criança necessitou ser encaminhada para monitorização em UTI ou precisou de oxigênio suplementar ou reintubação no período pós-operatório.

\section{DISCUSSÃO}

A indicação de AT é comum em crianças devido à hipertrofia das tonsilas podendo estar associada à AOS. A internação tende a ser breve, embora algumas 
crianças são mantidas internadas e em observação por alguma complicação no período imediato após a cirurgia ${ }^{17-20}$, sendo que a taxa de complicações varia de $2,6 \%, 5,8 \%$ à $8,8 \% 16,17,21$.

A população estudada apresentou distribuição por faixa etária com pico na idade entre 4 a 8 anos (média de 6,43), que corresponde ao período de maior prevalência de $\operatorname{AOS}^{1,2}$. Semelhante a literatura também observou-se predomínio do sexo masculino ${ }^{15,16}$.

As queixas mais frequentes elencadas pelos pais das crianças foram obstrução nasal, respiração oral e ronco, também concordando com os achados da literatura prévia $^{1,7,10,22}$. O ronco é apontado como importante preditor clínico a suspeita da AOS em crianças ${ }^{23,24}$. No presente estudo, o sintoma ronco esteve presente em toda a amostra, entretanto, ressalta-se que apenas o relato da sintomatologia não é eficaz para diagnosticar a AOS e diferenciar os graus de gravidade 1,20,21,23,24. A grande variedade de manifestações clínicas da AOS e a subjetividade do quadro clínico relatado pelos familiares contribui para não-homogeneidade dos sintomas em cada faixa etária, mesmo quando a severidade da síndrome é semelhante $2,23,24$.

Quanto à avaliação clínica otorrinolaringológica, estiveram presentes os aspectos de fácies adenoideana, palato em ogiva e mordida aberta ${ }^{25-27}$, sendo também apontados pela literatura em respiradores orais e crianças com AOS propriamente dita.

Com a realização da $\mathrm{AT}$, ocorre importante melhora da qualidade de vida e também dos sinas/sintomas originados pelo distúrbio obstrutivo respiratório na criança ${ }^{10,11,13,22,23,25}$. No presente estudo, foi verificada a frequência de AOS grave em $40 \%$ antes da cirurgia e de 10\% após o procedimento, constatando assim, a necessidade do acompanhamento longitudinal desses pacientes, não sendo unânime a melhora das crianças em toda a sua sintomatologia. Outros estudos já apontavam esta necessidade de acompanhamento, pois alguns parâmetros, como a respiração oral permanecia após a cirurgia, além de episódios de obstrução respiratória durante o sono ${ }^{28,29}$.

Além disso, a elevada frequência de apneias obstrutivas logo após a cirurgia também pode estar relacionada ao grau de comprometimento da resposta respiratória após anestesia e a resistência respiratória maior nas vias aéreas edemaciadas pela intubação orotraqueal ${ }^{15,16}$. Esta indução se faz pertinente, pois as crianças com AOS grave após AT, não correspondem aquelas constatadas previamente à cirurgia. Quanto aos fatores de risco para a AOS grave, houve a tendência para o grau da hipertrofia das tonsilas, tabagismo passivo, IVAS e fácies adenoideana. Esta tendência foi considerada 
clinicamente importante, pois considerou-se o pequeno número de participantes neste estudo piloto uma limitação importante. Sugere-se que novos estudos sejam realizados com amostras maiores, podendo-se então, melhorar o grau de evidência de correlação de fatores de riscos com a gravidade da AOS em crianças no pós-operatório.

Vale ressaltar que nenhuma criança em acompanhamento pós de AT na enfermaria pediátrica comum apresentou complicação respiratória maior com necessidade de transferência para unidade de terapia intensiva pediátrica. Desta forma, não é possível determinar se a gravidade da AOS teria sido um indicador confiável de complicações respiratórias.

\section{CONCLUSÃO}

A polissonografia demonstrou frequência elevada de SAOS grave em crianças em programação de cirurgia das tonsilas, até então subestimada. A persistência de sinais de gravidade de comprometimento respiratório é transitória, com melhora após 6 a 8 horas de cirurgia. Como possíveis fatores correlacionados, observou-se como tendência o grau de hiperplasia das tonsilas, infecções das VAS, tabagismo passivo e a presença de fácies adenoideano.

\section{REFERÊNCIAS}

1. Marcus CL, Brooks LJ, Draper KA, Gozal D, Halbower AC, Jones J, et al. Diagnosis and management of childhood obstructive sleep apnea syndrome. Pediatrics. 2012; 130(3): 576-84.

2. Li AM, Zhu Y, Au CT, Lee DL, Ho C, Wing YK. Natural history of primary snoring in school-aged children: a 4-year follow-up study. Chest. 2013; 143(3):729-35.

3. Cohen S, Springer C, Perles Z, Koplewitz BZ, Avital A, Revel-Vilk S. Cardiac, lung, and brain thrombosis in a child with obstructive sleep apnea. Pediatric. Pulmonol. 2010; 45 (8):836-9.

4. Kaditis AG, Alexopoulos El, Dalapascha M, Papageorgiou K, Kostadima E, Kaditis DG, et al. Cardiac systolic function in Greek children with obstructive sleep disordered breathing. Sleep Med. 2010; 11(4):406-12. 
5. Amin R, Somers VK, McConnell K, Willging P, Myer C, Sherman M, et al. Activityadjusted 24-hour ambulatory blood pressure and cardiac remodeling in children with sleep disordered breathing. Hypertension. 2008; 51(1):84-91.

6. Marcus CL, Carroll JL, Koerner CB, Hamer A, Lutz J, Loughlin GM. Determinants of growth in children with the obstructive sleep apnea syndrome. J Pediatr. 1994; 125:55662.

7. Tran KD, Nguyen CD, Weedon J, Goldstein NA. Child behavior and quality of life in pediatric obstructive sleep apnea. Arch Otolaryngol Head Neck Surg. 2005; 131(1):52-7.

8. Wu J, Gu M, Chen S, Chen W, Ni K, Xu H, Li X. Factors related to pediatric obstructive sleep apnea-hypopnea syndrome in children with attention deficit hyperactivity disorder in different age groups. Medicine (Baltimore). 2017; 96(42):e8281.

9. Gozal D. Sleep-disordered breathing and school performance in children. Pediatrics. 1998; 102(3 Pt 1):616-20.

10. Mitchell RB, Kelly J. Behavior, neurocognition and quality-of-life in children with sleep-disordered breathing. Int J Pediatr Otorhinolaryngol. 2006; 70(3):395-406.

11. Baugh RF, Archer SM, Rosenfeld RM, Amin R, Burns JJ, Darrow DH et al. Clinical practice guideline: tonsillectomy in children. Otolaryngol Head Neck Surg. 2011; 144(1S): S1-S30.

12. Bhattacharyya N. Ambulatory pediatric otolaryngologic procedures in the United States: characteristics and perioperative safety. Laryngoscope. 2010; 120(4):821-5.

13. Rosenfeld RM, Green RP. Tonsillectomy and adenoidectomy: changing trends. Ann Otol Rhinol Laryngol. 1990; 99(3 Pt 1):187-91.

14. Erickson BK, Larson DR, St Sauver JL, Meverden RA, Orvidas LJ. Changes in incidence and indications of tonsillectomy and adenotonsillectomy 1970-2005. Otolaryngol Head Neck Sug. 2009; 140(6):894-901.

15. Bonuck KA, Freeman K, Henderson J. Growth biomarker changes after adenotonsillectomy: systematic review and meta-analysis. Arch Dis Child. 2009; 94(2):83-91.

16. Brigger MT, Brietzke SE. Outpatient tonsillectomy in children: a systematic review. Otolaryngol Head Neck Surg. 2006; 135(1):1-7.

17. Martins RO, Castello-Branco N, Barros JL, Weber SA. Risk factors for respiratory complications after adenotonsillectomy in children with obstructive sleep apnea. J Bras Pneumol. 2015; 41(3):238-45. 
18. Bush B, Tobias JD, Lin C, Ruda J, Jatana KR, Essig G et al. Postoperative bradycardia following adenotonsillectomy in children: Does intraoperative administration of dexmedetomidine play a role? Int J Pediatr Otorhinolaryngol. 2018; 104:210-5.

19. Weber SA, Pierri Carvalho R, Ridley G, Williams K, El Dib R. A systematic review and meta-analysis of cohort studies of echocardiographic findings in OSA children after adenotonsilectomy. Int J Pediatr Otorhinolaryngol. 2014; 78(10):1571-8.

20. Jaryszak EM, Shah RK, Vanison CC, Lander L, Choi SS. Polysomnographic variables predictive of adverse respiratory events after pediatric adenotonsillectomy. Arch Otolaryngol Head Neck Surg. 2011; 137(1):15-8.

21. Saur JS, Brietzke SE. Polysomnography results versus clinical factors to predict postoperative respiratory complications following pediatric adenotonsillectomy. Int J Pediatr Otorhinolaryngol. 2017; 98:136-42.

22. Beraldin BS, Rayes TR, Villela PH, Ranieri DM. Avaliação do impacto da adenotonsilectomia sobre a qualidade de vida em crianças com hipertrofia das tonsilas palatinas e faríngeas. Rev Bras Otorrinolaringol. 2009; 75(1):64-9.

23. Tan HL, Alavarez MLA, Tsaoussoglou M, Weber SAT, Kaditis AG. When and why to treat the child who snores?. Pediatr Pulmonol. 2016; 52(3):399-412.

24. Kaditis AG, Alonso Alvarez ML, Boudewyns A, Alexopoulos El, Ersu R, Joosten K, et al. Obstructive sleep disordered breathing in 2- to 18-year-old children: diagnosis and management. Eur Respir J. 2016; 47(1):69-94.

25. Kawashima S, Peltomäki T, Sakata H, Mori K, Happonen R-P, Rönning O. Craniofacial morphology in preschool children with sleep-related breathing disorder and hypertrophy of tonsils. Acta Paediatr. 2002; 91:71-7.

26. Caprioglio A, Zucconi M, Calori G, Troiani V. Habitual snoring OSA and craniofacial modification. Orthodontic and diagnostic aspects in a case control study. Minerva Stomatol. 1999; 48:125-37.

27. Cappellette Jr M, Costa CMF, Besen A, Francisco SM, Souza HIP, Weckx LLM. Avaliação cefalométrica do padrão facial do respirador oral. Ortodontia. 2012; 45(3): 267-73.

28. Bueno D de A, Grechi TH, Trawitzki LV, Anselmo-Lima WT, Felício CM, Valera FC. Muscular and functional changes following adenotonsillectomy in children. Int J Pediatr Otorhinolaryngol. 2015; 79(4):537-40. 
29. Guilleminault C, Huang YS, Monteyrol PJ, Sato R, Quo S, Lin CH. Critical role of myofascial reeducation in pediatric sleep-disordered breathing. Sleep Med. 2013; 14(6):518-25.

Conflito de interesses: Os autores declaram não haver conflito de interesses.

Participação dos autores: Os autores declaram que participaram de todas as etapas do estudo (concepção, desenvolvimento do estudo, redação e revisão).

Como citar este artigo: Carvalho LP, Martins RO, Fonseca EL, Corrêa CC, Weber SAT. Identificação de fatores de risco para apneia obstrutiva do sono após adenotonsilectomia: estudo piloto. Journal Health NPEPS. 2018; $3(1): 132-142$.

Submissão: 23/01/2018

Aceito: 19/06/2018

Publicado: 30/06/2018 